\title{
Soft-Gluon Production Due to a Gluon Loop in a Constant Chromo-Electric Background Field
}

\author{
Gouranga C. Nayak ${ }^{1, *}$ and Peter van Nieuwenhuizen ${ }^{1, t}$ \\ 1 C. N. Yang Institute for Theoretical Physics, \\ Stony Brook University, SUNY, Stony Brook, NY 11794-3840, USA
}

(Dated: July 19, 2018)

\begin{abstract}
We obtain an exact result for the soft gluon production and its $p_{T}$ distribution due to a gluon loop in a constant chromo-electric background field $E^{a}$ with arbitrary color. Unlike Schwinger's result for $e^{+} e^{-}$pair production in QED which depends only on one gauge invariant quantity, the electric field $E$, we find that the $p_{T}$ distribution of the gluons depend on two gauge invariant quantities, $E^{a} E^{a}$ and $\left[d_{a b c} E^{a} E^{b} E^{c}\right]^{2}$.

PACS numbers: PACS: 11.15.-q, 11.15.Bt, 11.55.-m, 11.90.+t
\end{abstract}

*Electronic address: nayak@insti.physics.sunysb.edu

${ }^{\dagger}$ Electronic address: vannieu@insti.physics.sunysb.edu

Typeset by REVTEX 1 
Although hard gluon production at high energy colliders is computed by using perturbative quantum chromodynamics (pQCD), it is not possible to calculate soft gluon production by using perturbation theory because the coupling constant becomes large at low energy. In this paper we study non-perturbative soft gluon production due to vacuum polarization in a constant chromo-electric field by applying the background field method to QCD with gauge group $\mathrm{SU}(3)$. While $e^{+} e^{-}$production from a constant electromagnetic field in spinor QED has been calculated long ago by Euler and Heisenberg [1], and Schwinger [2], and by Weisskopf 3] for scalar QED, there is of course no direct photon production in QED as photons do not interact with a classical electromagnetic field. In the case of QCD, gluons do interact with a background chromofield and gluons are produced. As we want to study the $p_{T}$ distribution of gluons produced at collider experiments, we shall not employ Schwinger's proper time method but, rather, we will directly evaluate the path integral and obtain the partition function which gives both the $p_{T}$ distribution and the total gluon production.

For the interpretation of experiments which probe the quark-gluon plasma at high-energy large-hadron colliders such as RHIC (Au-Au collisions at $\sqrt{s}=200 \mathrm{GeV})[4]$ and LHC $(\mathrm{Pb}-\mathrm{Pb}$ collisions at $\sqrt{s}=5.5 \mathrm{TeV}$ ) [5] it might be necessary to know the $p_{T}$ spectrum of soft gluons produced by a chromo field. The physical picture behind this calculation is that two heavy nuclei collide and then move apart, creating a classical chromo field in between [6, 7]. We consider here a constant chromo-electric field along the beam direction. Subsequently this constant chromo-electric field breaks up into quark anti-quark pairs and gluons. The quark anti-quark production has been calculated before [8] and the corresponding $p_{T}$ distribution has been approximated by WKB methods [9]. Also the total gluon production rate by a covariantly constant field $\left(d N / d^{4} x\right)$ has been calculated for $\mathrm{SU}(2)$ in [10, 11] and for $\mathrm{SU}(3)$ in $[12]$. The $p_{T}$ distribution $\left(d N / d^{4} x d^{2} p_{T}\right)$ of gluon production due to a constant chromoelectric field which we present in this article is new, as far as we know, and we consider the realistic case of $\mathrm{SU}(3)$.

We obtain the following formula for the number of non-perturbative soft gluons produced per unit time and per unit volume and per unit transverse momentum from a given constant chromo-electric field $E^{a}$

$$
\frac{d N_{g g}}{d t d^{3} x d^{2} p_{T}}=\frac{1}{4 \pi^{3}} \sum_{j=1}^{3}\left|g \lambda_{j}\right| \ln \left[1+e^{-\frac{\pi p_{T}^{2}}{\left|g \lambda_{j}\right|}}\right] .
$$

This result is gauge invariant because $\left|\lambda_{1}\right|,\left|\lambda_{2}\right|$, and $\left|\lambda_{3}\right|$ are the positive square root of the 
following gauge invariant real positive quantities

$$
\begin{aligned}
\lambda_{1}^{2} & =\frac{C_{1}}{2}[1-\cos \theta] \\
\lambda_{2}^{2} & =\frac{C_{1}}{2}\left[1+\cos \left(\frac{\pi}{3}-\theta\right)\right], \\
\lambda_{3}^{2} & =\frac{C_{1}}{2}\left[1+\cos \left(\frac{\pi}{3}+\theta\right)\right],
\end{aligned}
$$

where $\theta$ is real and is given by

$$
\cos ^{3} \theta=-1+6 C_{2} / C_{1}^{3}
$$

They depend only on the Casimir invariants for SU(3)

$$
C_{1}=E^{a} E^{a}, \quad C_{2}=\left[d_{a b c} E^{a} E^{b} E^{c}\right]^{2},
$$

where $a, b, c=1, \ldots, 8$ are the color indices of the adjoint representation of the gauge group $\mathrm{SU}(3)$. Note that $\theta$ is real because $C_{1}^{3}-3 C_{2} \geq 0$.

This can be contrasted with the corresponding formula for massless fermion pair production

$$
\frac{d N_{e^{+} e^{-}}}{d t d^{3} x d^{2} p_{T}}=\frac{-|e E|}{4 \pi^{3}} \ln \left[1-e^{-\frac{\pi p_{T}^{2}}{|e E|}}\right] .
$$

The result for the $p_{T}$ distribution in (15) was obtained by WKB methods in [9] and integration over $p_{T}$ reproduces Schwinger's result for total production rate $d N / d^{4} x$ [2]. (The first term in the expansion of the logarithm of the total production rate $d N / d^{4} x$ was already obtained by Heisenberg and Euler [1]. For an excellent review on Euler-Heisenberg actions, see [13]). In our result the symmetric tensor $d_{a b c}$ appears. Hence the extension of Schwinger's formula for $e^{+} e^{-}$pair production to gluon production is not straight forward. We now present a derivation.

The Lagrangian density in the background field method of QCD with covariant background gauge fixing term in Feynman-'t Hooft gauge is given by [14]

$$
\mathcal{L}_{\text {gluon }}=\frac{1}{2} Q^{\mu a} M_{\mu \nu}^{a b}[A] Q^{\nu b}
$$

where

$$
M_{\mu \nu}^{a b}[A]=\eta_{\mu \nu}\left[D_{\rho}(A) D^{\rho}(A)\right]^{a b}-2 g f^{a b c} F_{\mu \nu}^{c}
$$


with $\eta_{\mu \nu}=(-1,+1,+1,+1)$ the Minkowski metric. The corresponding ghost Lagrangian density is given by

$$
\mathcal{L}_{\text {ghost }}=\bar{\chi}^{a} D_{\mu}^{a b}[A] D^{\mu, b c}[A+Q] \chi^{c}=\bar{\chi}^{a} K^{a b}[A, Q] \chi^{b}
$$

where $\mathrm{Q}, \mathrm{A}$ and $\chi$ are the gluon quantum field, the background field, and the ghost field, respectively, and $D_{\mu}[A] Q_{\nu}^{a}=\left[\partial_{\mu} Q_{\nu}^{a}+g f^{a b c} A_{\mu}^{b} Q_{\nu}^{c}\right]$.

The one-loop effective action for a gluon loop in a background field $A_{\mu}^{a}$ is given by

$$
S_{\text {gluon }}^{(1)}=-i \ln (\operatorname{Det} M)^{-1 / 2}=\frac{i}{2} \operatorname{Tr}[\ln M[A] H-\ln M[0] H]
$$

where $H^{\mu \nu}=\eta^{\mu \nu}$. We added the matrices $H^{\mu \nu}$ since this will allow us to factorize the trace over Lorentz indices. The trace $\operatorname{Tr}$ contains an integration over $d^{4} x$ and a sum over color and Lorentz indices. We replace the logarithm by using the exponential representation

$$
S_{\text {gluon }}^{(1)}=\frac{i}{2} \operatorname{Tr} \int_{0}^{\infty} \frac{d s}{s}\left[e^{i s(M[0] H+i \epsilon)}-e^{i s(M[A] H+i \epsilon)}\right] .
$$

We shall say more about the s-integration contour later. We assume that the electric field is along the z-axis (the beam direction) and we choose the gauge $A_{0}^{a}=0$ so that $A_{3}^{a}=-E^{a} \hat{x^{0}}$. The color indices $(a=1, \ldots .8)$ are arbitrary. Since $\Lambda^{a b}=i f^{a b c} E^{c}$ is hermitian and antisymmetric, its eigenvalues are real and come in pairs $(\lambda,-\lambda)$. We will show later that the matrix $\Lambda^{a b}$ has six non vanishing eigenvalues. So after diagonalization it reads

$$
\Lambda_{d}^{a b}=\left(\lambda_{1},-\lambda_{1}, \lambda_{2},-\lambda_{2}, \lambda_{3},-\lambda_{3}, 0,0\right) .
$$

We replace the derivative $\partial_{\mu}$ by $i p_{\mu}$ satisfying $\left[p_{\mu}, x^{\nu}\right]=-i \delta_{\mu}^{\nu}$ and obtain

$$
M[A]_{\mu \rho}^{a b} \eta^{\rho \nu}=\delta_{\mu}^{\nu}\left[\left({\hat{p_{0}}}^{2}-{\hat{p_{T}}}^{2}-{\hat{p_{3}}}^{2}\right) \delta^{a b}-2 g \Lambda^{a b}{\hat{x^{0}}}_{\hat{p_{3}}}-g^{2}\left(\Lambda^{2}\right)^{a b}{\hat{x^{0}}}^{2}\right]+2 i g \Lambda^{a b} S_{\mu}^{\nu}(12
$$

where $p_{T}=\sqrt{p_{1}^{2}+p_{2}^{2}}$ is the transverse momentum of the gluons (transverse to the electric field direction) and

$$
S_{\mu}^{\nu}=\left[\begin{array}{llll}
0 & 0 & 0 & 1 \\
0 & 0 & 0 & 0 \\
0 & 0 & 0 & 0 \\
1 & 0 & 0 & 0
\end{array}\right]
$$


To reduce this problem to one harmonic oscillator, we make the usual similarity transformation [15]. We also make a similarity transformation in group space which diagonalizes the matrices $\Lambda$

$$
M[A]_{\mu \rho}^{a b} \eta^{\rho \nu}=\left\{e^{i p^{3} p_{0} / g \Lambda_{d}}\left[\left({\hat{p_{0}}}^{2}-{\hat{p_{T}}}^{2}-g^{2}\left(\Lambda_{d}^{2}\right){\hat{x^{0}}}^{2}\right) \delta_{\mu}^{\nu}+2 i g \Lambda_{d} S_{\mu}^{\nu}\right] e^{-i p^{3} p_{0} / g \Lambda_{d}}\right\}^{a b} .
$$

Recalling that $\Lambda^{a b}$ has two vanishing eigenvalues, we should read this expression as follows: for $\mathrm{a}=\mathrm{b}=1, . .6$ each $\Lambda_{d}$ denotes an eigenvalue $\pm \lambda_{1}, \pm \lambda_{2} \pm \lambda_{3}$, while for $\mathrm{a}=\mathrm{b}=7,8$ one finds an expression which is independent of $A_{\mu}^{a}$ and which we omit because it cancels against the contributions from $M[0]$.

The trace over Lorentz indices factorizes because $\delta_{\mu}{ }^{\nu}$ commutes with $S_{\mu}{ }^{\nu}$ and we obtain

$$
\begin{aligned}
& S_{\text {gluon }}^{(1)}=\frac{-i}{2} \int_{0}^{\infty} \frac{d s}{s} \sum_{j=1}^{6} \operatorname{tr}\left[e^{i p^{3} p_{0} / g \lambda_{j}} e^{i s\left({\hat{p_{0}}}^{2}-{\hat{p_{T}}}^{2}-g^{2} \lambda_{j}^{2}{\hat{x_{0}}}^{2}+i \epsilon\right)} e^{-i p^{3} p_{0} / g \lambda_{j}}\right. \\
& \left.\left[2+2 \cosh 2 s g \lambda_{j}\right]-4 e^{i s\left({\hat{p_{0}}}^{2}-{\hat{p_{T}}}^{2}+i \epsilon\right)}\right]
\end{aligned}
$$

The trace tr denotes integral over a complete set of $\mathrm{x}$ eigenstates. From here we proceed as in QED [15]. We add complete sets of p eigenstates, and obtain

$$
S_{\text {gluon }}^{(1)}=\frac{-i}{2} \int_{0}^{\infty} \frac{d s}{s} \sum_{j=1}^{6} \frac{1}{(2 \pi)^{3}} \int d^{4} x \int d^{2} p_{T} e^{-i s p_{T}^{2}-s \epsilon}\left[\left|g \lambda_{j}\right| \frac{1+\cosh 2 s g \lambda_{j}}{\sinh s\left|g \lambda_{j}\right|}-\frac{2}{s}\right] .(16
$$

The one-loop effective action for the ghost in the background field $A_{\mu}^{a}$ is given by

$$
S_{\text {ghost }}^{(1)}=-i \ln (\operatorname{Det} K)=-i \operatorname{Tr} \int_{0}^{\infty} \frac{d s}{s}\left[e^{i s[K[0]+i \epsilon]}-e^{i s[K[A]+i \epsilon]}\right]
$$

where $K^{a b}[A]$ is given by (8). Since there is now no trace over $\mu, \nu$ but also no square root of the ghost determinant, we find an overall factor $-1 / 2$. In addition there is no term $f^{a b c} F_{\mu \nu}^{c}$ in $K[A]$ so that the term $\cosh 2 s g \lambda_{j}$ is absent. We obtain then

$$
S_{\text {ghost }}^{(1)}=\frac{i}{2} \int_{0}^{\infty} \frac{d s}{s} \sum_{j=1}^{6} \frac{1}{(2 \pi)^{3}} \int d^{4} x \int d^{2} p_{T} e^{-i s p_{T}^{2}-s \epsilon}\left[\left|g \lambda_{j}\right| \frac{1}{\sinh s\left|g \lambda_{j}\right|}-\frac{1}{s}\right] .
$$

Adding the effective action due to a gluon and a ghost loop we find for the one-loop effective action

$$
S^{(1)}=\frac{-i}{2} \int_{0}^{\infty} \frac{d s}{s} \sum_{j=1}^{6} \frac{1}{(2 \pi)^{3}} \int d^{4} x \int d^{2} p_{T} e^{-i s p_{T}^{2}-s \epsilon}\left[\left|g \lambda_{j}\right| \frac{\cosh 2 \operatorname{sg} \lambda_{j}}{\sinh s\left|g \lambda_{j}\right|}-\frac{1}{s}\right] .
$$

The s-integral at fixed $p_{T}$ is convergent at $\mathrm{s} \rightarrow 0$, but integration over $p_{T}$ yields an extra factor $1 / \mathrm{s}$, and charge renormalization cures this ultraviolet problem by substracting also 
the term linear in $s$ in the expansion of $\frac{\cosh 2 s\left|g \lambda_{j}\right|}{\operatorname{sinhs}\left|g \lambda_{j}\right|}$. In fact the term linear in s yields the beta function [10, 11].

A more serious problem occurs for $\mathrm{s} \rightarrow \infty$. Obviously, the integral over $\mathrm{s}$ diverges, indicating the presence of infrared divergences. Although such divergences in Feynman graphs with on-shell external gluons are well known, the full non-perturbative treatment of these divergences seems complicated. However, we are only interested in the imaginary part of the effective action, since it gives us the gluon production rate, and hence we proceed similarly to Yildiz and Cox (see eq. 22 in [12]). We use the well-known expansion

$$
1 / \sinh x=\frac{1}{x}+2 x \sum_{n=1}^{\infty} \frac{(-1)^{n}}{x^{2}+n^{2} \pi^{2}}
$$

and then we formally replace $s$ by -is (as first advocated by Schwinger in QED where no infrared problems are present). The integral is now real, except for half-circles around the poles at $s\left|g \lambda_{j}\right|=-i n \pi$ for $\mathrm{n}=1,2,3 \ldots$ The term $1 / x$ in (20) cancels against the term $1 / s$ in (19). This yields probability for gluon production per unit time and per unit volume

$$
W_{\text {gluon }}=2 \operatorname{Im} S^{(1)}=\frac{1}{(2 \pi)^{3}} \int d^{2} p_{T} \sum_{n=1}^{\infty}(-1)^{n+1} \sum_{j=1}^{6} \frac{\left|g \lambda_{j}\right|}{n} e^{\left[-\frac{n \pi p_{T}^{2}}{\mid g \lambda_{j}}\right]} .
$$

All that is left is to determine the eigenvalues $\lambda_{j}(\mathrm{j}=1, \ldots, 8)$ of the matrix $i f^{a b c} E^{c}$. First we evaluate the determinant of the $8 \times 8$ matrix $\left[f^{a b c} E^{c}-\lambda \delta^{a b}\right]$ and find

$$
\begin{aligned}
\operatorname{Det}\left[f^{a b c} E^{c}-\lambda \delta^{a b}\right]=\quad & \lambda^{2}\left[\lambda^{6}+A \lambda^{4}+B \lambda^{2}+C\right] \\
& =\lambda^{2}\left(\lambda^{2}+\lambda_{1}^{2}\right)\left(\lambda^{2}+\lambda_{2}^{2}\right)\left(\lambda^{2}+\lambda_{3}^{2}\right) .
\end{aligned}
$$

Because $E^{b}$ is an eigenvector of the matrix $i f^{a b c} E^{c}$, and because eigenvalues come in pairs $\left(\lambda_{j},-\lambda_{j}\right)$, there are two eigenvalues zero, which explains the factor $\lambda^{2}$. The gauge invariant quantities $\mathrm{A}, \mathrm{B}$, and $\mathrm{C}$ in the above equation can only depend on the Casimir invariants in (4I) and we find

$$
A=\frac{3}{2} E^{a} E^{a}, \quad B=\frac{A^{2}}{4}, \quad C=\frac{1}{16}\left(\left[E^{a} E^{a}\right]^{3}-3\left[d_{a b c} E^{a} E^{b} E^{c}\right]^{2}\right),
$$

where $d_{a b c}$ is the symmetric invariant tensor in the adjoint representations of $\mathrm{SU}(3)$. It follows that the three eigenvalues satisfy

$$
\begin{aligned}
& \lambda_{1}^{2}+\lambda_{2}^{2}+\lambda_{3}^{2}=A \\
& \lambda_{1}^{2} \lambda_{2}^{2}+\lambda_{2}^{2} \lambda_{3}^{2}+\lambda_{3}^{2} \lambda_{1}^{2}=B \\
& \lambda_{1}^{2} \lambda_{2}^{2} \lambda_{3}^{2}=C,
\end{aligned}
$$


the solution of which is given by eq. (2).

In this letter we have derived the $p_{T}$ distribution of the soft gluons produced by the vacuum polarization due to a gluon loop in a constant chromo-electric field. We have used the background field method of QCD with gauge group SU(3). For application at RHIC and LHC we have constructed the $p_{T}$ distribution of the gluon production. We find that, unlike the case in $\mathrm{QED}$ where the $e^{+} e^{-}$pair production rate depends on one gauge invariant quantity $|E|$, the $p_{T}$ distribution of the gluon production rate depends on two gauge invariant quantities, $E^{a} E^{a}$ and $\left[d_{a b c} E^{a} E^{b} E^{c}\right]^{2}$. We intend to add a chromo-magnetic field later.

\section{Acknowledgments}

We thank Fred Cooper, Gerald Dunne, Martin Rocek, Jack Smith, George Sterman and William Weisberger for discussions. This work was supported in part by the National Science Foundation, grants PHY-0098527, PHY-0354776 and PHY-0354822.

[1] W. Heisenberg and H. Euler, Z. Physik 98, (1936) 714.

[2] J. Schwinger, Phys. Rev. 82 (1951) 664.

[3] V. Weisskopf, Kong. Dans. Vid. Selsk. Math-fys. Medd. XIV No. 6 (1936); English translation in: Early Quantum Electrodynamics: A Source Book, A. I. Miller, (Cambridge University Press, 1994).

[4] L. McLerran and M. Gyulassy, Nucl. Phys. A750 (2005) 30.

[5] Y. Schutz, J. Phys. G30 (2004) S903.

[6] G. Baym, Phys. Lett. B138 (1984) 18; K. Kajantie and T. Matsui, Phys. Lett. B164 (1985) 373; K. J. Eskola and M. Gyulassy, Phys. Rev. C47 (1996) 2329; G. C. Nayak and V. Ravishankar, Phys. Rev. D55 (1996) 6877; Phys. Rev. C58 (1998) 356; R. S. Bhalerao and G. C. Nayak, Phys. Rev. C61 (2000) 054907; Y. Kluger, J. M. Eisenberg, B. Svetitsky, F. Cooper, E. Mottola, Phys. Rev. Lett. 67 (1991) 2427; Phys. Rev. D45 (1992) 4659; D. Kharzeev and K. Tuchin, hep-ph/0501234. (Quark-antiquark production using Schwinger's approach).

[7] L. McLerran and R. Venugopalan Phys. Rev.D50 (1994) 2225; D. Dietrich, G. C. Nayak and W. Greiner, Phys. Rev. D64 (2001) 074006; M. Gyulassy and L. Mclerran, Phys. Rev. C56 
(1997) 2219; D. Dietrich, Phys. Rev. D70 (2004) 105009. (Perturbative calculations involving a space-time dependent classical color field).

[8] M. Claudson, A. Yildiz and P. H. Cox, Phys. Rev. D22 (1980) 2022.

[9] A. Casher, H. Neuberger and S. Nussinov, Phys. Rev. D20 (1979) 179.

[10] S. G. Matinyan and G. K. Savvidy, Nucl. Phys. B134 (1978) 539.

[11] H-T Sato, M. G. Schmidt and C. Zahlten, hep-th/0003070, M. Reuter, M. G. Schmidt and C. Schubert, Ann. Phys. 259 (1997) 313.

[12] A. Yildiz and P. H. Cox, Phys. Rev. D21 (1980) 1095.

[13] G. V. Dunne, Heisenberg-Euler effective Lagrangians: Basics and extensions, hep-th/0406216, to appear in the Memorial Volume for Ian Kogan.

[14] G. 't Hooft, Nucl. Phys. B62 (1973) 444.

[15] C. Itzykson and J-B. Zuber, Quantum Field Theory, Mc-Graw Hill (1980) page 193; L. AlvarezGaume and E. Witten, Nucl. Phys. B234 (1984) 234; P. van Nieuwenhuizen, Anomalies in Quantum Field Theory: Cancellation of Anamolies in $d=10$ Supergravity, Lecture Notes in Mathematical and Theoretical Physics, Vol 3, Leuven University Press (1988), page 46. 